\title{
ANÁLISIS DE VARIABLES PSICOLÓGICAS ASOCIADAS A LA SALUD Y PRODUCTIVIDAD EN LA UNIVERSIDAD
}

\section{ANALYSIS OF PSYCHOLOGICAL VARIABLES ASSOCIATED WITH THE HEALTH AND PRODUCTIVITY IN UNIVERSITY}

\section{PABLO RUISOTO', SILVIA LIBERTAD VACA ${ }^{2}$, MARCO A. JIMÉNEZ², ROCÍO RAMÍREZ², JHON ESPINOSA²}

\author{
Investigador Prometeo de la SENESCYT, Universidad Técnica Particular de Loja (Ecuador). Universidad de Salamanca (España). \\ ruisoto@usal.es \\ 2 Universidad Técnica Particular de Loja (Ecuador)
}

El consumo de sustancias adictivas y los problemas de ansiedad y depresión constituyen algunos de las complicaciones de la salud más frecuentes en la población, con serios costes para su bienestar y la productividad de la comunidad o institución a la que pertenecen. Se presenta un proyecto dirigido a analizar la distribución del estrés percibido y variables que modulan su impacto sobre la salud de la comunidad universitaria, incluyendo inflexibilidad psicológica y compromiso vital, predictores de bienestar psicológico. Se seleccionaron variables y escalas breves y adecuadas psicométricamente para la evaluación online anónima de profesores, estudiantes y personal de administración y servicios. Las variables seleccionadas fueron: estrés psicológico y variables mediadoras de su impacto sobre el consumo de sustancias y la salud: apoyo social, personalidad y estilos de afrontamiento. Los indicadores criterio de salud fueron: niveles de ansiedad y depresión, consumo de sustancias y satisfacción vital y laboral. El proyecto permitirá obtener perfiles que reflejen la distribución de las variables analizadas en la comunidad universitaria. Se discuten las implicaciones de los resultados esperados en el diseño y priorización de intervenciones dirigidas a la promoción de la salud, bienestar y productividad de la comunidad universitaria.

PALABRAS CLAVE: estrés, salud, consumo de sustancias, ansiedad, depresión, inflexibilidad psicológica, compromiso vital, comunidad universitaria.
Both substance abuse and anxiety/depression disorders are among the most common health complications with serious costs for welfare and productivity of the community or institution to which they belong. This study presents a project aimed to analyze the distribution of perceived stress and variables that modulate its impact on the health at the university community, including life-engagement, psychological inflexibility and predictors of psychological well-being. Key variables and scales for their measurement were carefully selected according to their psychometric properties and application time for the online and anonymous evaluation of faculty, students and administrative staff and services. Target variables were the following: psychological stress, social support, personality and coping styles. The following health indicators were: levels of anxiety and depression, substance consumption, and life/job satisfaction. The project will provide profiles regarding the distribution of analyzed variables in the university community. Implications of those profiles for the design and prioritization of interventions aimed to promote health, welfare and productivity of the university community are further discussed.

KEYWORDS: stress, health, substance abuse, anxiety, depression, psychological inflexibility, life-engagement, universitary community. 
INTRODUCCIÓN

La Organización Mundial de la Salud (OMS) define salud mental como "estado de bienestar en el que cada individuo es consciente de su potencial, puede responder apropiadamente a los estresores diarios de la vida, trabajar productiva y fructíferamente, ser capaz de hacer una contribución a su comunidad" (WHO, 2013). Paradójicamente, el incremento en los niveles de estrés psicológico (Cohen, Janicki-Deverts, 2012), el aumento en el consumo de alcohol y otras sustancias (OMS, 2014) y el incremento en la incidencia de ansiedad y depresión en la población general (OMS, 2014), reflejan una situación alejada del ideal salud y bienestar buscados.

El estrés se ha considerado como uno de los principales factores responsables del deterioro de múltiples indicadores de salud y bienestar de las personas (Sapolsky, 2004; Marmot, 2010; Hill, 2013; Allen, Balfour, Bell, Marmot, 2014). Puede expresarse que la percepción psicológica de una situación continuada de estrés caracterizada por la percepción de falta de control y predictibilidad sobre los resultados de nuestro comportamiento, está asociada con dos efectos directamente relacionados con la salud: 1) incremento del riesgo de problemas de ansiedad y depresión (Arborelius, Owens, Plotsky, Nemeroff, 1999; Holsboer, 2000; McQuade, Young, 2000; Krishnadas, Cavanagh, 2012; Marmot, 2004) y 2) incremento del consumo de alcohol y otras sustancias (Deroche, Gamonet, Sillaber, Aouizerate, Izawa, Jaber, et al., 2003; Sinha, 2008) e incluso la reducción del éxito de intentos de abandonar el consumo de tabaco (Cohen, Lichtenstein, 1990; Mermelstein, Cohen, Lichtenstein, Baer, Kamark, 1986; Cohen, 2000). El consumo de alcohol por si solo está asociado con mayor riesgo, con la violencia, depresión, suicidio, complicaciones físicas y la pérdida de hasta 4 años de vida (OMS, 2014).

El estrés también supone complicaciones a nivel del sistema inmunitario, enlenteciendo los procesos de curación, disminuyendo la respuesta inmune a vacunas, aumentando la vulnerabilidad ante agentes infecciosos y aumentando las posibilidades de reactivación de virus latentes (Herbert, Cohen, Miller, 2001; Cohen, 2005; Glaser, Kiecolt-Claser, 2005; Sagerstroom, Miller, 2007); a nivel cardiovascular, aumentando el riesgo de accidentes cardiovasculares y cerebrovasculares (Friedman, Rosenman, 1974; Meerson, 1994; Pickering, 2004; Janicki-Deverts, Cohen, Matthews,
Cullen, 2008; Zucchi, Kirkland, Jadavji, van Waes, Klein, Supina, Metz, 2009); a nivel metabólico incrementando el riesgo de diabetes tipo 2 y el riesgo de desarrollar el síndrome metabólico (Cohen, Manuck, 1995; Surwit, Williams, 1996; Vitaliano, Scanlan, Zhang, Savage, Hirsch, Siegler, 2002); a nivel gastrointestinal, aumentando el riesgo de úlceras y síndrome del colon irritable (Brady, Porter, Conrad, Mason, 1958; Bennett, Tennant, Piesse, 1998; Mayer, 2000) e incluso en qué y cuánto comer (Dallman, Prcoraro, Akana, Fleur, Gómez, Houshyar, Bhatnagar, Laurgero, Manalo, 2003; Adam, Epel, 2007).

El impacto negativo del estrés sobre la salud parece estar mediado por, al menos, tres variables: 1) un estilo de personalidad caracterizado por la tendencia a la competitividad, hostilidad y presión del tiempo, denominado tipo A, que supondría mayor vulnerabilidad al estrés (Friedman, Rosenman, 1974; Haynes, Baker, 1982; Johnston, 1986; Cohen, Matthews, 1987; Cole, Kawachi, Liu, Gaziano, Manson, Buring, Hennekens, 2001; Williams, Nieto, Sanford, Couper, Tyroler, 2002; Marmot, Bruner, 2005); 2) la soledad o percepción de falta de apoyo social ((Hawkley, Burleson, Bertoson, Cacioppo, 2003; Golden, Conroy, Bruce, et al, 2009), también asociado a mayor consumo de sustancias (Cohens, Matthews, 1978; Cohen, Wills, 1985; Lepore, Alen, Evans, 1993; Orth-Gomer, Wamala, Horsten, Schenck-Gustafsson, Schneiderman, Mittleman, 2000; Pressman, Cohen, Miller, Barkin, Treanor, 2005); 3) el estilo de afrontamiento caracterizado por un control aversivo, principalmente conductas de evitación y escape, asociado al término de inflexibilidad psicológica, lo que supondría un mayor riesgo para el desarrollo de casi cualquier problema psicológico, a diferencia de un estilo de afrontamiento caracterizado por la aceptación de eventos internos aversivos y compromiso con los valores, considerado un factor protector (Hayes, Kapust, Leonard, Rosenfarb, 1981; Hayes, Wilson, Gifford, Follette, 1996; Vilardaga, Estévez, Levin, \& Hayes, 2012) y próximo al concepto tradicional de eudemonia (Ryan, Deci, 2001; Lubomirsky, King, Diener, 2005).

En Ecuador, la salud se considera una prioridad; pero la mayor parte de los estudios se han dirigido al estudio del consumo de sustancias (CONSEP, 2013; Bravo Orti, Palucci Marziale, 2010) en personal de administración y servicios (Bravo Orti, Palucci Marziale, 2010), profesores de ciencias sociales (Sierra, 2012) o estudiantes 
(Consejo Nacional de Control de Sustancias Estupefacientes y Psicotrópicas, 2013). Sin embargo, hasta nuestro conocimiento, no existe ningún estudio dirigido a evaluar una comunidad universitaria completa de forma integral e incluyendo variables asociadas al estrés, por lo que la información disponible es insuficiente para ofrecer una visión global de la comunidad universitaria y poder trasladar dicha información en intervenciones concretas, especialmente, considerando la estrecha relación entre estrés en el contexto laboral y la salud (Rossberg, Eiring, Friis, 2004; Faragher, Cass, Cooper, 2005; Quintero, Biela, Barrera, Campo, 2007).

El objetivo de este trabajo es revisar y seleccionar las variables psicológicas con mayor impacto sobre la salud y productividad de los diferentes segmentos de la comunidad universitaria.

\section{MATERIALES Y MÉTODOS}

El análisis de las variables psicológicas relacionadas con la salud y la productividad; así como la selección de las escalas estandarizadas a utilizar para su evaluación en este estudio se realizó tomando en consideración los artículos publicados en los últimos 10 años en MedLine y aquellos especialmente relevantes para el campo.

Las variables seleccionadas fueron estrés psicológico y variables mediadoras de su impacto sobre el consumo de sustancias y la salud: apoyo social, personalidad y estilos de afrontamiento.

Los indicadores criterio de salud fueron niveles de ansiedad y depresión, consumo de sustancias y satisfacción vital y laboral, las mismas que serán evaluadas a través de una encuesta tipo online a docentes, estudiantes de pregrado, administrativos y personal de servicio de la Universidad Técnica particular de Loja.

El estudio ha sido aprobado por el

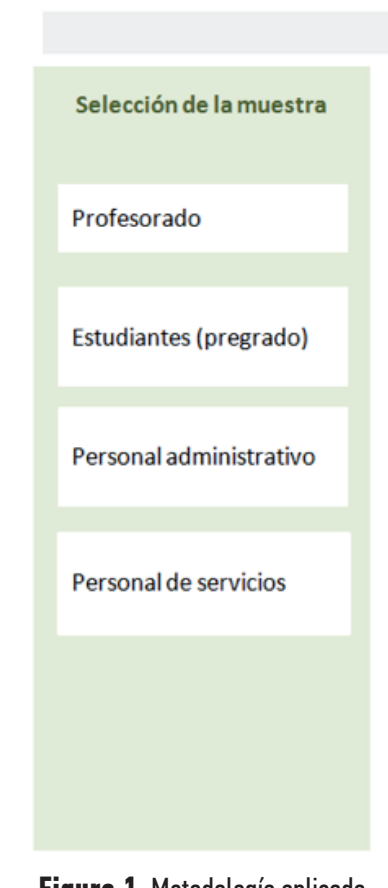

Figura 1. Metodología aplicada. comité ético local y se desarrollará de acuerdo con las normas de la Declaración de Helsinki.

El acceso de los docentes y estudiantes se realizaría a través del Entorno Virtual de Aprendizaje (EVA), garantizando su pertenencia a la comunidad universitaria.

Posteriormente se solicitaría la aceptación de un consentimiento informado donde se detallarían los objetivos el estudio, el carácter anónimo de sus respuestas e imposibilidad de vincular éstas con los datos identificativos, la utilización con fines de investigación de los datos, el carácter voluntario, y la garantía de obtener un informe personalizado con sus resultados (sin referencia alguna a datos personales) de forma gratuita e inmediata. Una vez finalizada la aplicación se registrarían las respuestas en cada una de las escalas; así como el cuestionario sociodemográfico y el tiempo en segundos de aplicación de la prueba que serían inmediatamente exportados a una hoja de datos en formato excel y alojado en el mismo servidor que la encuesta.

Los participantes cuyo tiempo de aplicación fuera inferior a 7 minutos (50\% del tiempo medio requerido para completar la prueba en base al pilotaje realizado), serían automáticamente descartados.

Se realizarán análisis descriptivos y de varianza multivariadas, así como pruebas de t-Student para el análisis de diferencias utilizando SPSS v. 21 para Mac. Ver Figura 1.

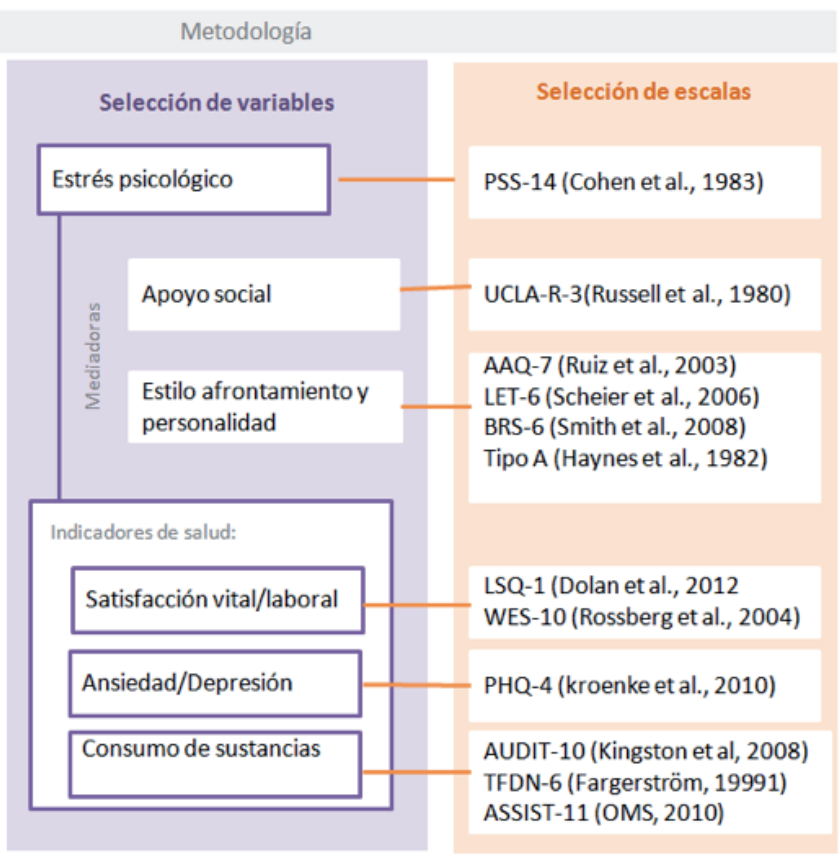


RESULTADOS

Las variables e instrumentos seleccionados para la inclusión en el estudio propuesto son las siguientes:

Cuestionario para la evaluación de datos sociodemográficos: sexo, edad, ocupación, lugar de residencia, tiempo de bajas laborales/ausencia clases. Se incluye una versión simplificada del The Income Evaluation Question (IEQ) (Van Praag and Kapteyn, 1971) para la evaluación de la percepción de pobreza; y el Índice de Masa Corporal (IMC) o índice de Quetelet (Keys, Fidanza, Karvonen, Kimura, Taylor, Henry, 1972) para la evaluación del peso deseable según estatura a partir de la fórmula IMC = masa $(\mathrm{Kg})$ I [altura (m) x altura (m)].

Perceived Stress Scale (PSS-14). (Cohen, Kamarck, y Mermelstein, 1983; versión en español de Remor, Carrobles, 2001; Remor, 2006). Es el instrumento psicológico más empleado en la evaluación de la percepción de estrés actual. Evalúa el grado en el que las situaciones de la vida de una persona son consideradas como estresantes, es decir, cómo valoran sus vidas en términos de impredecibilidad, incontrolabilidad y sobrecarga. Los sujetos responden en una escala de 0 (nunca) a 4 (siempre). Un ejemplo de ítem sería: "Con qué frecuencia te has sentido mal por cosas que ocurrieron inesperadamente?"

UCLA Loneliness Scale Revised-Short (Hughes, Waite, Hawkley, Cacioppo, 2004). Se utilizó la versión breve compuesta por los ítems 2, 11 y 14 de la escala original (Russel, Peplau, Cutrona, 1980 con adaptación española de Morejon, García-Boveda, 1994) diseñada para evaluar la sensación subjetiva de soledad entendida como la percepción de una menor disponibilidad de relaciones significativas de la deseada, especialmente referida al apoyo emocional. Los sujetos puntúan cada ítem como 0 ("Nunca me siento así1"), 1 ("Pocas veces me siento así”), 2 (“En ocasiones me siento as"), 3 ("con frecuencia me siento así"). Un ítem de ejemplo sería: "¿Con qué frecuencia se siente dejado de lado?”.

Type A Behavior Scale (Haynes, Baker, 1982). Escala de 9 ítems empleado en el estudio clásico de Framingham. Evalúa personalidad definida por un complejo acción-emoción caracterizada por tendencia a la competitividad, intensa motivación de logro, hostilidad, agresividad, impaciencia y un sentido de urgencia exagerado, y que se han encontrado asociadas con problemas de salud, incluyendo trastornos cardiovascula- res y muerte prematura. Los sujetos responden Un ejemplo de ítem sería: "Tener una fuerte necesidad de sobresalir (ser el mejor) en la mayoría de las cosas".

Avoidance and Action Questionnaire (AAQ7) (Ruíz, Langer, Luciano, Cangas, Beltrán, 2013). Constituye la medida general más utilizada de flexibilidad/inflexibilidad psicológica y consta de 7 ítems, basados en el original (Hayes, et al., 2004). El participante responde en una escala tipo Likert de 7 puntos, siendo $1=$ nunca es verdad y $7=$ siempre es verdad. Un ejemplo de ítem sería: "Tengo miedo de no poder controlar mis preocupaciones y sentimientos”.

Life Engagement Test (Scheier, Wrosch, Baum, Cohen, Martire, Matthews, Schulz, Zdaniuk, 2006). Se trata de una escala de 6 ítems diseñado para medir compromiso vital, entendido como el grado en el que la persona está comprometido en actividades que son valiosas para la persona. Un ejemplo de ítem sería: "Para mí, las cosas que hago todas merecen la pena". Los sujetos responden en una escala tipo Likert siendo $1=$ muy en desacuerdo, $2=$ en desacuerdo, 3 = neutral, 4 = de acuerdo, $5=$ muy de acuerdo.

Brief Resilience Scale (BRS) (Smith, Dalen, Wiggins, Tooley, Christopher, Bernard, 2008). Consta de 6 ítems dirigidos a evaluar el grado resiliencia o capacidad de las personas de adaptarse al estrés y situaciones adversas. Los participantes responden en una escala tipo Likert de 5 puntos, siendo 1 = muy en desacuerdo y 5 = muy de acuerdo. Un ítem de ejemplo sería: "Tiendo a recuperarme rápidamente después de momentos difíciles".

Alcohol Use Disorders Identification Test (AUDIT-C) (Self-report version) (Kriston, Hölzel, Weiser, et al. 2008). Se utilizó la versión modificada de 3 ítems del AUDIT original (Babor, Higgins-Biddle, Monteiro, 2001) diseñados para la evaluación del consumo problemático de alcohol. El sujeto responde eligiendo entre 5 opciones de respuesta correspondientes a la frecuencia del enunciado y que oscilan de "nunca" a "a diario o casi a diario". Un ítem de ejemplo sería: "En el último año con ¿con qué frecuencia consumes una bebida que contiene alcohol?".

Test de Fagerström (Fagerström, 1978; Heatherton, Kozlowski, Freker, Fagerström, 1991). Se utilizó la versión revisada de 6 ítems de la versión original () dirigido para evaluar el consumo problemático de tabaco. El formato de respuesta diferente para cada ítem. Un ítem de ejemplo sería: "Cuántos cigarrillos fumas al día”. 
The Alcohol, Smoking and Substance Involvement Screening Test (ASSIST) (OMS, 2010). Se aplicó el ítem 2 relativo la consumo de sustancias en los últimos tres meses.

Patient Health Questionnaire of Depression and Anxiety (PHQ-4) (Kroenke, Sitzer, Williams, Löwe, 2009; Wingenfeld, Schneider, Brähler, 2010). Consiste en 4 ítems dirigidos a evaluar depresión y ansiedad asociados con deterioro social y bajas. El participante responde en una escala de $0=$ "nunca" a 3 = "casi diariamente" en base a cómo se han sentido en las últimas dos semanas. Un ítem de ejemplo sería: "no soy capaz de o controlar mis preocupaciones".

Satisfaction Life Question (LSQ) (Dolan, Layard, Metcalfe,2011; Helliwell, Layard, Sachs, 2012). Se utilizó la siguiente pregunta del The World Health Organization Quality of Life (WHOQOL)-BREF (2004), European Social Survey y World Happiness Report (2012) para evaluar satisfacción vital: "Actualmente, ¿cuál es su grado de satisfacción con su vida en un conjunto“.El sujeto responde en una escala de 0 = extremadamente insatisfecho a $10=$ extrema damente satisfecho

Working Environment Scale-10 (WES-10) (Friis, 1981;Rossberg, Eiring, Friis, 2004). Consta de 10 ítems autoadministrados que evalúa el clima o satisfacción laboral. Los sujetos responden en una escala de 5 opciones de respuesta que varían de nunca a casi siempre y se puntúan de 0 a 4 respectivamente, de forma que las puntuaciones oscilan entre 0 y 40 puntos. No hay punto de corte y mayor puntuación indica mayor grado de satisfacción laboral. Un ejemplo ítem sería: "¿Consideras que te imponen demasiadas tareas o cosas que hacer en un día”.

En cuanto al procedimiento previsto para la recogida de datos, todos los participantes deberán completar un consentimiento informado subrayando el anonimato de los datos para reducir la probabilidad de deseabilidad social y en cumplimiento

En la aplicación de las escalas, previa aprobación por el comité ético local y de acuerdo con los principios de la Declaración de Helsinki, se utilizarían traducciones de las versiones originales publicadas en inglés yo/ castellano (España) al idioma nativo de la muestra (castellano en Ecuador), se enfatizará la equivalencia cros-cultural y conceptual frente a la equivalencia lingüística o literal de acuerdo con las directrices de la OMS, incluyendo los métodos de: traducción directa, panel de expertos para traducción inversa, aplicación de prueba y versión final (http://www.who.int/substance_abuse/research_tools/translation/en/).

El análisis de datos incluirá estadísticos descriptivos, cálculos de diferencias de media pruebas t-de Student, análisis de varianza y análisis multivariados para el análisis, por ejemplo de diferencias de género en las variables registradas. Todos los análisis serán realizados utilizando SPSS 21 para Mac.

A nivel individual, cada uno de los participantes recibirá un breve informe describiendo los resultados obtenidos en las pruebas incluidas.

A nivel grupal, se obtendrán 1) perfil relativo a la distribución del consumo de alcohol y otras sustancias psicoactivas; 2) perfil relativo a la distribución de estrés psicológico así como variables mediadoras del impacto del estrés sobre la salud, incluyendo el consumo de sustancias, como son: el estilo de personalidad tipo A, apoyo social, y estilos de afrontamiento problemático y saludable. 3) erfil relativo a la distribución de problemas de ansiedad, depresión y bienestar o satisfacción subjetivo.

Complementariamente, se podrían realizar análisis detallados del comportamiento de cada una de las variables en los grupos analizados delimitados por las variables sociodemográficas evaluadas.

\section{DISCUSIÓN}

Los resultados de la aplicación de este estudio facilitan el diagnóstico de necesidades en relación con las variables que suponen mayor vulnerabilidad de la población estudiada ante problemas relacionados con el consumo de sustancias y salud en general. Supone por lo tanto, un punto de comienzo para la priorización y desarrollo de intervenciones que actúen sobre las variables analizadas y maximicen las oportunidades de desarrollar una vida plena y satisfactoria en la línea de los esfuerzos realizados en los últimos años (Rodgers, 1979; North, Syme, Feeney, Shipley, Marmot, 1996; Kawachi, Kennedy, Lochner, Prothrow-Stith, 1997; Kawachi, Kennedy, 2002; Marmot, 2004; Steptoe, Kunz-Ebrecht, Owen, Feldman, Willemsen, Kirschbaum, Marmot, 2004; Stockwell, Auld, Zhan, Martin, 2012). Uno de los beneficios derivados de planes de intervención más eficientes será la mejora de los niveles de productividad e innovación en la universidad, reduciendo innecesarias y evitables bajas labo- 
rales que, sólo en Reino Unido, han supuesto un coste de más de 1600 millones de USD para el contribuyente (Black, 2008; Marmot, Bruner, 2005; Marmot, 2012).

En consonancia con la literatura, dos serían los aspectos prioritarios a intervenir. Por un lado, actuaciones dirigidas a reducir el estrés psicológico, de acuerdo con los hallazgos de la Comisión sobre Determinantes Sociales de la Salud de la Organización Mundial de la Salud (OMS), que subrayan su importancia, no como un concepto abstracto; sino un indicador de salud pública tan significativo como las tasas de consumo de tabaco, obesidad y actividad física. Sin duda, intervenciones en este sentido reducirán el riesgo asociados a la aparición y agravamiento de problemas relacionados con el consumo de sustancias y otros problemas de salud relacionados directamente con la salud y cada vez más frecuentes en la sociedad actual. Por otro lado, el actuaciones dirigidas a abordar la inflexibilidad psicológica, de acuerdo con los hallazgos de las terapias contextuales, y en particular, de la terapia de aceptación y compromiso (ACT) (Hayes, Levin, 2012; Luoma, Drake, Hayes, Kohlenberg, 2011; Wilson, Hayes, 2000; Wilson, Hayes, Byrd, 2000). Un constructo relativamente nuevo pero asociado con múltiples problemas psicológicos, incluyendo el consumo problemático de sustancias, donde resulta obvio el papel que desempeña la evitación experiencial, o consumo dirigido a control o eliminar pensamientos, sensaciones y sentimientos no deseados, siendo la intolerancia al malestar y la tendencia a suprimir pensamientos predictores ya conocidos del consumo problemático de sustancias y del fracaso en los tratamientos. Desde ACT, las intervenciones están explícitamente dirigidas a incrementar la flexibilidad psicológica, y han mostrado resultados positivos en consumo de sustancias como el alcohol (Heffner, Eifert, Parker, Hernández, Sperry, 2003) y otras sustancias (Twohig, Shoenberger, Hayes, 2007; Smout, et al., 2010; Hayes, Wilson, 2004).

En una segunda fase de este estudio se propone la replicación de este estudio en otras comunidades universitarias de Ecuador, con la finalidad de realizar un diagnóstico de necesidades en la comunidad universitaria a nivel nacional, y por extensión, contribuir a la priorización y diseño de intervenciones más eficientes en el contexto de las prioridades del Plan del Buen Vivir en Ecuador.

\section{CONCLUSIONES}

En este trabajo se describe con detalle el proceso seguido en 1) la selección de variables psicológicas asociadas con múltiples indicadores de salud, incluyendo una revisión exhaustiva de la literatura científica en psicología de la salud y psicología clínica; 2) la selección de escalas breves que cumplan los criterios psicométricos requeridos por la comunidad científica para garantizar la obtención de indicadores cuantitativos fiables y válidos, y permitan su integración en un protocolo único; 3) el diseño de un sistema digitalizado de recogida de datos que permita accede a toda la comunidad universitaria, recogida automática de datos en una hoja de datos para su análisis estadístico inmediato; 4) la identificación de necesidades y prioridades de intervención de acuerdo a los perfiles obtenidos por los diferentes segmentos de la comunidad universitaria.

\section{AGRADECIMIENTOS}

El presente trabajo fue financiado por la Universidad Técnica Particular de Loja, el Proyecto Prometeo de la Secretaría de Educación Superior, Ciencia, Tecnología e Innovación y el Observatorio Nacional de Drogas del Consejo Superior para el Control de Sustancias Estupefacientes de la República del Ecuador.

\section{REFERENCIAS BIBLIOGRÁFICAS}

Adam, T.C., Epel, E.S. (2007): Stress, eating and the reward system. Physiology and Behavior, 91, 449-458.

Allen, J., Balfour, R., Bell, R., Marmot, M. (2014): Social determinants of mental health. International Review of Psychiatry, 26(4), 392-407.

Arborelius, L., Owens, M.J., Plotsky, P.M., Nemeroff, C.B. (1999): The role of corticotropin-releasing factor in depression and anxiety disorders. Journal of Endocrinology, 160, 1-12.

Babor, T.F., Higgins-Biddle, J.C., Monteiro, M.G. (2001): Audit. The Alcohol Use Disorders identification Test. World Health Organization.

Bennett, E., tennant, C., Piesse, C. (1988): Level of chronic life stress predicts clinical outcome in irritable bowel syndrome. Gut, 43, 256.

Black, D.C. (2008): Working for a healthier tomorrow. Review of the health of Britain's working age population. London: TSO (The Stationery Office)

Brady, J. Porter, D., Conrad, D., Mason, J. (1958): Avoidance behavior and the development of gastroduodenal ulcers. Journal of Experimental Analysis of Behavior, I, 69. 
Bravo Orti, C.M., Palucci Marziale, M.H. (2010): El consumo de alcohol en personal administrativo y de servicios de una universidad del Ecuador. Revista Latina Enfermagem, 18, 487-495

Cohen, S., Janicki-Deverts, D. (2012): Who's Stressed? Distributions of Psychological Stress in the United States in Probability Samples from 1983, 2006, and 2009, Journal of applied social psychology,42, 1320-1334.

Cohen, S., Kamarck, T., Mermelstein, R. (1983): A global measure of perceived stress. Journal of Health and Social Behavior, 24, 385-396.

Cohen, S., Lichtenstein, E. (1990). Perceived Stress, Quitting Smoking, and Smoking Relapse. Health Psychology, 9(4), 466-478.

Cohen, S., Manuck, S.B. (1995): Stress, Reactivity, and Disease, Psychosomatic Medicine, 57(5), 423-426.

Cohen, S., Matthews, K.A. (1987): Social support, Type A Behavior, and coronary artery disease. Psychosomatic Medicine, 49, 425-330.

Cohen, S., Miller, G.E. (2001): Psychological stress and antibody response to immunization: a critical review to the human literature, Psychosomatic Medicine, 63(7), 7-18.

Cohen, S., Wills, T. (1985): Stress, social support and the buffering hypothesis. Psychological Bulletin, 98, 310.

Cole, S.R., Kawachi, I., Liu, S., Gaziano, J.M., Manson, J.A.E., Buring, J.E., Hennekens, C.H. (2001): Time urgency and risk of non-fatal myocardial infarction. International Journal of Epidemiology, 30, 363-369.

Consejo Nacional de Control de Sustancias Estupefacientes y Psicotrópicas (2013): Informe de Investigación sobre uso de dorgas en estudiantes de la Universidad Nacional de Loja.on

Dallman, M.F., Prcoraro, N., Akana, F., Fleur, S.E., Gómez, F., Houshyar, H., Bhatnagar, M.E. S., Laurgero, K.D., Manalo, S. (2003): Chronic stress and obesity: A new view of "comfort food". Proceedings of the National Academy of Sciences, 100(20), 11696-11701.

Deroche,-Gamonet, V., Sillaber, I., Aouizerate, B.,Izawa, R., Jaber, M., Ghozland, S., Kellendonk, C., Le Moal, M., Spanagel, R., Schutz, G., Tronche, F, Piazza, P.V. (2003): The glucocorticoid receptor as a potential target to reduce cocaine abuse. Journal of Neuroscience, 23, 4785.

Faragher EB, Cass M, Cooper CL. (2005): The relationship between job satisfaction and health: a meta-analysis. Occup Environ Med.; 105-112.

Friedman, M., Rosenman, R. (1974): Type A Behavior and Your Heart. New York:
Glaser, R., Kiecolt-Glaser, J.K. (2005): Stress-induced immune dysfunction: Implications for health. Nature Reviews Immunology, 5, 243-251.

Golden J, Conroy RM, Bruce I, et al (2009): Loneliness, social supports, mood and wellbeing in community-dwelling elderly, International Journal of Geriatric Psychiatry, 24, 694-700.

Hayes, S.C., Kapust, J. Leonard, S.R., Rosenfarb, I. (1981): Escape from freedom: choosing not to choose in pigeons. Journal of the Experimental Analysis of Behavior, 36(7), 1-7

Hayes, S.C., Levin, M. (Eds.). (2012): Mindfulness and Acceptance for Addictive Behaviors: Applying Contextual CBT to Substance Abuse and Behavioral Addictions. New Harbinger Publications.

Hayes, S.C., Strosahal, K., Wilson, K.G., Bisssett, R.T., Pistorello, J., Toarmino, D., .... Niccolls, R. (2004): Measuring experiential avoidance: A preliminary test of a working model. The Psychlogical Record, 54, 553-578.

Hayes, S.C., Wilson, K.G., Gifford, E.V., Bisset, R., Piasecki, M., Batten, S.V., ..., Gregg, j. (2004): A preliminary trial of twelve-step facilitation and acceptance and commitment therapy with polysubstance-abusing methadone-maintained opiate addicts. Behavior Therapy, 35, 667-688.

Hayes, S.C., Wilson, K.G., Gifford, E.V., Follette, V.M. (1996): Experiential Avoidance and Behavioral Disorders: A Functional Dimensional Approach to Diagnosis and Treatment. Journal of Consulting and Clinical Psychology, 64(6), 1152-1168.

Haynes, S.G., Baker, E.E. (1982): Type A behavior and the ten year incidence of coronary heart disease in the Framinhgam Heart Study. Activitas Nervosa Superior, 3(1), 57-77.

Hawkley, L.C., Burleson, M.H., Berntson, G.G., Cacioppo, J.T. (2003): Loneliness in Everyday Life: Cardiovascular Activity, Psychosocial Context, and Health Behaviors. Journal of Personality and Social Psychology, 85, 105-120

Heatherton, T.F., Kozlowski, L. T., Frecker, R.C., Fagerström, K.O. (1991): The Fagerstrom Test for Nicotine Dependence: a revision of the Fagerstrom Tolerance Questionnaire. British Journal of Addiction, 86, 1119-1127.

Heffner, M., Eifert, G.H., Parker, B.T., Hernández, D.H., Sperry, J.A. (2003): Valued directions: Acceptance and commitment therapy in the treatment of alcohol dependence. Cognitive and Behavioral Practice, 10, 378-383.

Helliwell, J. Layard, R., Sachs, J. (2012): World Happiness Report. Retrieved from http://www. earth.columbia.edu/sitefiles/file/Sachs $\% 20$ 
Writing/2012/World\%20Happiness\%20Report. pdf

Herbert, T., Cohen, S. (1993): Stress and immunity in humans: a meta-analytic review. Psychosomatic Medicine, 55, 364.

Hill, V. (2013): Theories of Stress and Its Relationship to Health, 22-42. Acceso el 3 de octubre de 2013 desde http://www.sagepub.com/upmdata/44175_2.pdf

Holsboer, F. (2000): The corticosteroid receptor hypothesis of depression. Neuropsychopharmacology, 23, 477.

Hughes, M.E., Waite, L.J., Hawkley, L.C., Cacioppo, J.T. (2004): A Short Scale for Measuring Loneliness in Large Surveys. Research in Aging. 26(6), 655-672.

Janicki-Deverts, D., Cohen, E., Matthews, K.A., Cullen, M.R. (2008): History of Unemployment Predicts Future Elevations in C-Reactive Protein among Male Participants in the Coronary Artery Risk Development in Young Adults (CARDIA). Study, Annals of Behavioral Medicine, 36, 176-185.

Johnston, D.W. (1986): Can and should Type A behaviour be changed? Postgraduate Medical Journal, 62, 785-788

Kawachi, I., Kennedy, B.P., Lochner, K., ProthrowStith, D. (1997): Social capital, income inequality, and mortality. American Journal of Public Health, 87(9), 1491-1498.

Kawachi, I Kennedy, B. (2002): The Health of Nations: why inequality is harmful to your health. New York: New York Press.

Keys, Ancel; Fidanza, Flaminio; Karvonen, Martti J.; Kimura, Noboru; Taylor, Henry L. (1972): Indexes of relative weight and obesity. Journal of Chronic Diseases, 25 (6-7), 329-343.

Krishnadas, R., Cavanagh, J. (2012): Depression: an inflammatory illness? Journal of Neurology, Neurosurgery and Psychiatry, 83, 495-502.

Kriston, L., Hölzel, L., Weiser, A.K., et al. (2008): Meta-analysis: are 3 questions enough to detect unhealthy alcohol use?. Annals of Internal Medicine, 149(12), 879-888.

Kroenke, K., Spitzer, R.L., Williams, J.B., Löwe, B. (2009): An ultra-brief screening scale for anxiety and depression: the PHQ-4. Psychosomatics, 50(6), 813-621. doi: 10.1176/appi.psy.50.6.613.

Lepore, S., Alen, K., Evans, G. (1993): Social support lowers cardiovascular reactivity to an acute stressor. Psychosomatic Medicine, 55, 518.

Marmot, M, Bruner, E. (2005): Cohort Profile: The Whitehall II study. International Journal of Epidemiology, 34, 251-256
Marmot, M. (2012): Why should the rich care about the health of the poor? Canadian Medical American Journal, 184(11), 1231-1232.

Marmot, M. (2010): Fair Society, Healthy Lives. The Marmot Review. Strategic Review of Health Inequalities in England.

Marmot, M. et al. (2004): Work, Stress and Health: The White Hall Study II.1-28.

Mayer, E. (2000). The neurobiology of stress and gastrointestinal disease. Gut, 47, 861.

McQuade, R., Young, A.H.Y. (2000): Future therapeutic targets in mood disorders: the glucocorticoid receptor. The British Journal of Psychiatry, 177, 390-395.

Meerson, F. Z. (1994): Stress-Induce Arrhythmic Disease of the Heart. Part I. Clinical Cardiology, 17, 362-371.

Mermelstein, R., Cohen, S., Lichtenstein, E., Baer, J.S, Kamark, T. (1986): Social support and smoking cessation and maintenance. Journal of consulting and clinical psychology, 54(4), 447453.

Morejon, A. J. V., Garcia-Boveda, R. J. (1994): RULS: Escala de soledad UCLA revisada. Fiabilidad y validez de una versión española. Revista de Psicología de la Salud, 6(1), 45-54

North, F.M., Syme, L.S., Feeney, A., Shipley, M., Marmot, M. (1996): Psychosocial Work Environment and Sickness Absence among British Civil Servants: The Whitehall II Study. American Journal of Public Health, 86, 332-340.

OMS (2010). The Alcohol, Smoking and Substance Involvement Screening Test (ASSIST): manual for use in primary care”. Retrieved from http:// www.who.int/substance_abuse/activities/ assist_screening_spanish.pdf?ua $=1$

OMS (2014): Global status report on alcohol and health. Individual Country Profiles. Recuperado de http://www.who.int/substance_abuse/ publications/global_alcohol_report/profiles/ecu. pdf?ua=1 el 21 de agosto de 2014.

Orth-Gomer, K., Wamala, S.P., Horsten, M., Schenck-Gustafsson, K., Schneiderman, N., Mittleman, M.A. (2000): Marital stress worsens prognosis in women with coronary heart disease. The Stockholm Female Coronary Risk Study. Journal of American Association of Medicine, 284(23), 3008-3014.

Pickering, T.G. (2004): Work and Blood Pressure. The Journal of Medical Hypertension, 6(7), 403-305.

Pressman, S.D., Cohen, S., Miller, G.E., Barkin, A., Treanor, J.J. (2005): Loneliness, social network size and immune response to influenza vaccination in college freshman, Health Psychology, 24(3), 297-306. 
Quintero, I.L. , Biela, R., Barrera, A., Campo, A.A. (2007): Exploratory factorial analysis of the working environment scale (WES) among employees of a public psychiatric hospital in Bucaramanga, Colombia. Revista Facultad de Medicina de la Universidad de Colombia, 55, 2430.

Remor, E. \& Carrobles, J.A. (2001): Versión Española de la escala de estrés percibido (PSS-14): Estudio psicométrico en una muestra VIH+. Ansiedad y Estrés, 7 (2-3), 195-201.

Remor, E. (2006): Psychometric Properties of a European Spanish Version of the Perceived Stress Scale (PSS). The Spanish Journal of Psychology, 9 (1), 86-93.

Rodgers, G.B. (1979): Income and inequality as determinants of mortality: an international crosssection analysis, Population Studies, 33, 343.

Rossberg, J.I., Eiring, O., Friis, S. (2004): Work environmentandjobsatisfaction. Apsychometric evaluation of the Working Environment Scale-10. Social Psychiatry and Psychiatric Epidemiology, 39, 576-580.

Ruíz, F.J., Langer, A.I., Luciano, C., Cangas, A.J., Beltrán, I. (2013). Measuring experiential avoidance and psychological inflexibility: The Spanish Version of the Acceptance and Action Questionaire-II. Psicothema, 25(1), 123-129.

Ryan, R.M., Deci, E.L. (2001): On happiness and human potentials: a review of research on hedonic and eudaimonic wellbeing. Annual Review of Psychology, 2, 141-166

Sapolsky, R.M. (2004): Why zebras don't get ulcers.3rd ed. Holt Paperbacks

Scheier, M.F., Wrosch, C., Baum, A., Cohen, S., Martire, L.M., Matthews, K.A., Schulz, R., Zdaniuk, B. (2006). The Life Engagement Test: Assessing Purpose in Life. Journal of Behavioral Medicine, 29(3), 291-298.

Sinha, R. (2008). Chronic Stress, Drug Use, and Vulnerability to Addiction. Annual New York Academy of Sciences, 1141, 105-1130.

Smith, B.W., Dalen, J., Wiggins, K., Tooley, E., Christopher, P., Bernard, J. (2008): The Brief Resilience Scale: Assessing the Ability to Bounce Back. International Journal of Behavioral Medicine, 15, 194-200.

Smout, M., Lngo, M., Harrison, S., Minniti, R., Wices, W., White, J. (2010). Psychosocial treatment for methamphetamine use disorders: A preliminar randomized controlled trial of cognitive behavior therapy and acceptance and commitment therapy. Substance Abuse, 31, 98107.

Steptoe, A., Kunz-Ebrecht, S., Owen, N., Feldman, P., Willemsen, G., Kirschbaum, C., Marmot, M.
(2004). Socioeconomic status and stress-related biological responses over the working day, Psychosomatic Medicine.

Surwit, R.W., Williams, P.G. (1996): Animal Models Provide Insight Into Psychosomatic Factors in Diabetes. Psychosomatic Medicine, 58, 582-589.

Twohig, M.P., Shoenberger, D., Hayes, S.C. (2007): A preliminary investigaction of acceptance and commitment therapy as a treatment for marijuana dependence in adults. Journal of Applied Behavior Analysis, 40, 619-632.

Vitaliano, P.P., Scanlan, J.M., Zhang, K., Savage, M.V., Hirsch, I.B., Siegler, I.C. (2002). A path model of chronic stress, the metabolic syndrome, and coronary heart disease. Psychosomatic Medicine, 64(3), 418-435.

Wilson, K. G. \& Hayes, S. C. (2000): Why it is crucial to understand thinking and feeling: an analysis and application to drug abuse. The Behavior Analyst, 23, 25-43.

Wilson, K. G., Hayes, S. C., \& Byrd, M. (2000): Exploring compatibilities between Acceptance and Commitment Therapy and 12-Step treatment for substance abuse. Journal of Rational-Emotive and Cognitive-Behavior Therapy, 18, 209-234.

Williams, J., Nieto, F., Sanford, C., Couper, D., Tyroler, H. (2002): The association between trait anger and incident stroke risk. Stroke 33, 13.

Wingenfeld, K., Schneider, A., Brähler, E. (2010): A 4-item measure of depression and anxiety: Validation and standardization of the Patient Health Questionnaire-4 (PHQ-4) in the general population. Journal of Affective Disorders, 122, 86-95.

Zucchi, F.C.R., Kirkland, S.W., Jadavji, N.M., van Waes, L.T., Klein, A., Supina, R.D., Metz, G.A. (2009): Predictable stress versus unpredictable stress: A comparison in a rodent model of stroke. Behavioral Brain Research,

Van Praag, Bernard M. S. (1971): The Welfare Function of Income in Belgium: An Empirical Investigation. European Economic Review, 2(3), 337-69.

Vilardaga, R., Estévez, A., Levin, M. E., \& Hayes, S. C. (2012): Deictic relational responding, empathy and experiential avoidance as predictors of social anhedonia: Further contributions from relational frame theory. The Psychological Record, 62, 409-432.

FIGURA 1: Representación gráfica de los aspectos metodológicos básicos: segmentos de la comunidad universitara a incluir en lamuestra, selección de variables variables clave a: estrés, variables mediadoras e indicadores de salud; y escalas para la obtención de indicadores cuantitativos. 